\title{
Exploring the fundamental limits of planar antennas using optimization techniques
}

\author{
Morteza Shahpari, David V. Thiel, Andrew Lewis \\ Griffith University, Brisbane, Australia \\ m.shahpari@griffith.edu.au,d.thiel@griffith.edu.au, a.lewis@griffith.edu.au
}

\begin{abstract}
New lower physical bounds on the quality factor $Q$ were recently reported in the literature by Yaghjian et al, and Mohammadpour et al. Due to the approximations made in derivation of the limits, examination of the limits for different antennas is very important but has not been reported. In this paper, physical bounds are examined for a set of meander line optimized antennas with different resonant frequencies. $Q$ factor simulation results compare well with the limiting $Q$ values.
\end{abstract}

\section{INTRODUCTION}

Fundamental limits on the performance of electrically small antennas is a useful guidline for design engineers to assess the merits of different designs. The first fundamental limit was develped by Wheeler [1] and Chu [2] to estimate the lowest achievable $Q$ factor in a fixed antenna volume. The Chu limit was later modified by McLean [3]. Investigations on the Chu-McLean limit shows that this limit has been valid for almost all the published designs in the literature [4]. Despite the validity of the limit, the Chu-McLean limit is far lower than the practical performance of antennas. Novel approaches has been introduced recently to estimate the minimum $Q$ in the fixed volume [5]-[9].

Initially, Gustafsson et al [5] used the forward scattering sum rule to derive a bound on $D / Q$ where $D$ is antenna directivity. Later Yaghjian and Stuart [6] made quasi-static approximations to calculate stored energy and radiated power and derived $Q$. Works in [5], [6] proposed the limit on the arbitrary antenna geometries. Mohammadpour-Aghdam et al [7] started from the results in [6] and derived a bound on planar antennas. They introduced a figure of merit (FOM) in order to compare the $Q$ factor of designed antenna and the minimum achievable $Q$ for a planar structure.

Ant colony optimisation (ACO) is a promising technique to optimize meander line antennas [10]. A set of meander line antennas (different shapes and resonant frequencies) was optimized in terms of highest radiation efficiency and lowest resonant frequency by ACO method. Optimized antennas were used to study the bounds on planar antennas. These meander line antennas are constructed in $5 \times 10$ rectangular grids.

In this paper, the limits by [6], [7] are studied for a set of optimized meander line antennas. The simulated $Q$ factor for 9 different antennas is compared with the limits proposed by [6], [7] and bounding $Q_{C h u}$.

\section{BOUNDS ON $Q$ FACTOR}

Chu [2] used an equivalent circuit for the characteristic impedance of $T E$ and $T M$ modes to calculate radiated power and stored energy. The Chu-McLean's limit is written as:

$$
Q_{C h u}=\frac{1}{k a}+\frac{1}{(k a)^{3}}
$$

where $k, a$ are the wave number and the radius of the circumscribing sphere, respectively. The Chu-McLean limit is popular due to its simplicity (only dependent on $k a$ ) and validity.

Using quasi-static approximations, Yaghjian and Stuart [6] derived the bound on $Q$ which is a function of polarizability ${ }^{1}$ $\gamma$ and volume $V$ of antenna.

$$
Q_{Y, l b}=\frac{6 \pi}{k^{3} \gamma}(1-V / \gamma)
$$

Considering the fact that the volume of the planar antennas is negligible, Mohammadpour Aghdam et al [7] wrote (2) in the following form:

$$
Q_{\text {rect }, l b}=\frac{9 / 2}{(k a)^{3}} \frac{1}{\gamma_{l}^{n}}
$$

where $\gamma_{l}^{n}$ is the normalized polarizability of the rectangle. This can be calculated as a function of lenght to width ratio $\xi$ [7]

$$
\gamma_{l}^{n}=\frac{0.29 \xi^{3}+4.6 \xi^{2}+0.3 \xi-0.11}{\xi^{3}+3.8 \xi^{2}-1.5 \xi+3.9}
$$

$Q$ in (3) is the lowest possible value for a planar structure. Figure of merit (FOM) [7] can be used as a tool to distinguish performance of outstanding planar antennas.

$$
F O M=\frac{Q_{\text {Ant }}}{Q_{\text {rect }, l b}}
$$

\section{RESULTS AND DISCUSSION}

To calculate the bound by Yaghjian and Stuart [6], the polarizability of each antenna was calculated directly using a method of moments solver. The polarizability $\gamma$ and volume $V$ was substituted in (2).

Different bounds on $Q$ are illustrated in Fig.1. As expected, the Chu-McLean limit is the lowest bound. Bounds proposed by Yaghjian and Stuart [6] and Mohammadpour-Aghdam et al [7] are very close together for a range of electrical length $k a$. As seen in Fig.1, the $Q$ of the optimized antennas follow the bounds in the range of $k a>0.5$, however it increases significantly for the antennas with $k a<0.5$.

\footnotetext{
${ }^{1}$ Polarizability was denoted with $\alpha$ in [6], but we use $\gamma$ because of consistency with other recent publications
} 


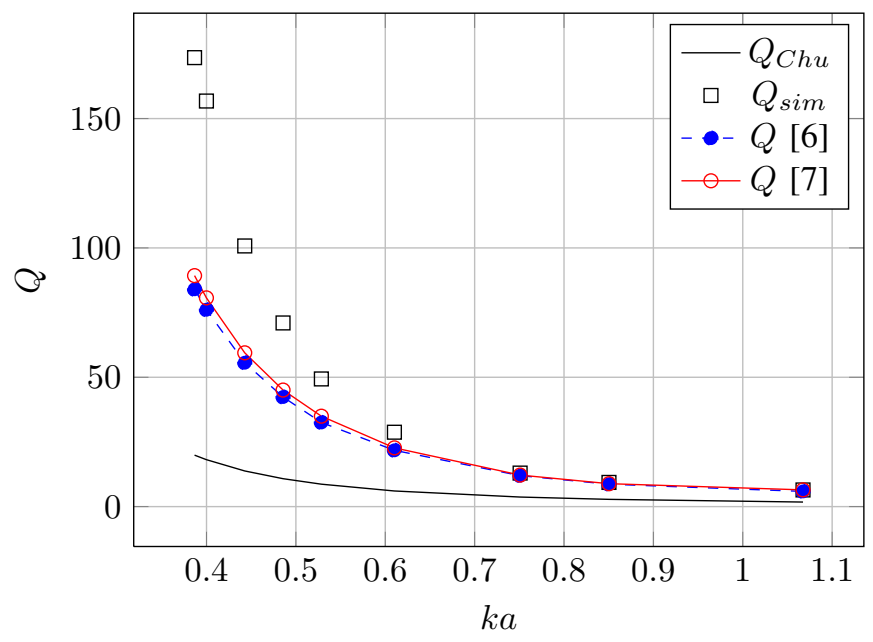

Fig. 1. Comparison of simulated $Q$ and different limiting $Q$

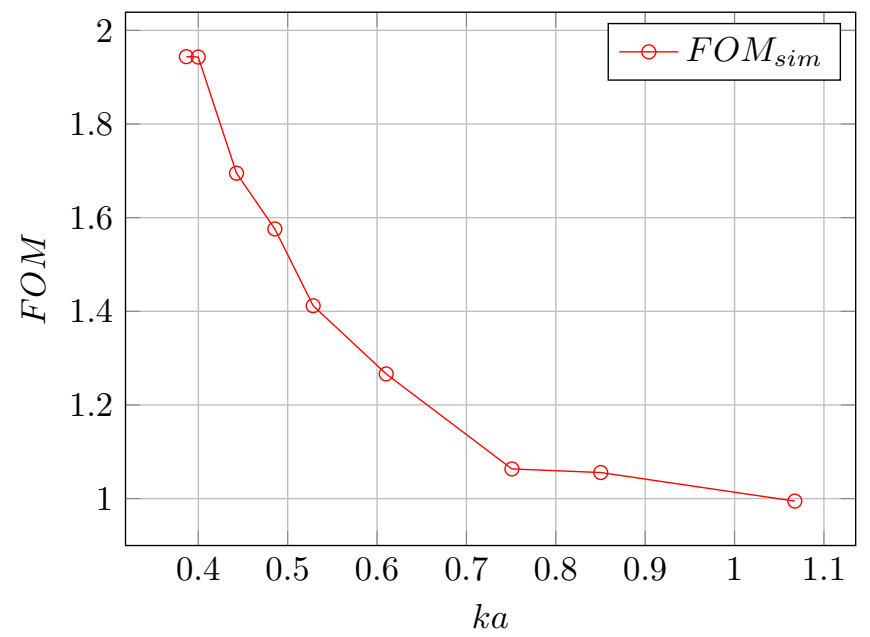

Fig. 2. FOM versus $k a$ for designs in [7] and 9 optimized planar antennas

Figure2 shows figure of merit of meander line antennas according to different $k a$ values. Meander line antennas are less efficient in terms of $\mathrm{Q}$ and bandwidth rather ideal case when $k a$ approaches zero. Actually this is the price that one needs to pay when decreases antenna dimensions.

\section{CONCLUSION}

This work has demonstrated the validity of the limits proposed by [6] and [7] using meanderline antennas optimized for large efficiency and less resonant frequency.

\section{REFERENCES}

[1] H. Wheeler, "Fundamental limitations of small antennas," Proc. IRE, vol. 35, no. 12, pp. 1479 - 1484, Dec. 1947.

[2] L. J. Chu, "Physical limitations of omni-directional antennas," J. Appl. Phys., vol. 19, no. 12, pp. 1163-1175, 1948. [Online]. Available: http://link.aip.org/link/?JAP/19/1163/1

[3] J. S. McLean, "A re-examination of the fundamental limits on the radiation Q of electrically small antennas," IEEE Trans. Antennas Propag., vol. 44, no. 5, p. 672, May 1996.
[4] D. Sievenpiper, D. Dawson, M. Jacob, T. Kanar, S. Kim, J. Long, and R. Quarfoth, "Experimental validation of performance limits and design guidelines for small antennas," IEEE Trans. Antennas Propag., vol. 60, no. 1, pp. $8-19$, Jan. 2012.

[5] M. Gustafsson, C. Sohl, and G. Kristensson, "Illustrations of new physical bounds on linearly polarized antennas," IEEE Trans. Antennas Propag., vol. 57, no. 5, pp. 1319 -1327, May 2009.

[6] A. Yaghjian and H. Stuart, "Lower bounds on the q of electrically small dipole antennas," IEEE Trans. Antennas Propag., vol. 58, no. 10, pp. $3114-3121$, Oct. 2010.

[7] K. Mohammadpour-Aghdam, R. Faraji-Dana, G. Vandenbosch, S. Radiom, and G. Gielen, "Physical bound on Q factor for planar antennas," in European Microwave Conference (EuMC), Oct. 2011.

[8] G. Vandenbosch, "Simple procedure to derive lower bounds for radiation of electrically small devices of arbitrary topology," IEEE Trans. Antennas Propag., vol. 59, no. 6, pp. 2217 -2225, June 2011.

[9] J. Chalas, K. Sertel, and J. Volakis, "Q limits for arbitrary shape antennas using characteristic modes," in European Conference on Antennas and Propagation (EUCAP), March 2012.

[10] A. Lewis, M. Randall, A. Galehdar, D. Thiel, and G. Weis, "Using ant colony optimisation to construct meander-line RFID antennas," in Biologically-Inspired Optimisation Methods, A. Lewis, S. Mostaghim, and M. Randall, Eds. Springer Berlin Heidelberg, 2009. 